Rev. Elev. Méd. vét. Pays trop., 1979, 32 (4) : 401-407.

\title{
Pouvoir d'établissement de quatre graminées fourragères tropicales (Pennisetum purpureum Schum., Panicum maximum Jacq., Brachiaria decumbens Stapf. et Digitaria decumbens Stent.) installées en arrière-saison
}

\author{
par K. G. KPAKOTE $(*)$, J. B. VILLARES $\left({ }^{*}\right)$ et G. P. ROCHA $\left({ }^{*}\right)$
}

RÉSUMÉ

\begin{abstract}
Les auteurs exposent les résultats qu'ils ont obtenus, en installant, en fin de saison des pluies, quatre graminées fourragères (Pennisetum purpureum Schum., Panicum maximum Jacq., Brachiaria decumbens Stapf. et Digitaria decumbens Stent.), sur un terrain pauvre situé dans la région de Botucatu, Etat de São Paulo, au Brésil. Les a prises 》 des boutures ont été excellentes, sauf pour Digitaria qu a eu à souffnr d'une invasion de cochenilles; et les productions en fourrage, au cours de la saison des pluies suivante, ont été très satisfaisantes.

Cette technique mise en pratique derrière une culture vivrière saisonnière avec ce que cela comporte d'avantages pour les plantes bouturées devrait donner des résultats d'un intérêt économique.
\end{abstract}

\section{INTRODUCTION}

Alors que, dans les pays développés, l'herbe fourragère passe au rang de vraie culture, et l'utilisation d'espèces fourragères sélectionnées se trouve répandue, dans les pays en développement d'une façon générale les aliments du bétail consistent essentiellement encore en herbes poussant naturellement en savanes et dont l'unique moyen de contrôle demeure le feu.

L'un des freins essentiels au développement de la production de quantités suffisantes d'herbes de bonne valeur nutritive dans nos régions résiderait, sans doute, dans les contraintes de coût de production des fourrages face au prix de la viande sur les marchés. En effet, le coût

(*) Ecole Supérieure d'Agronomie, Université du Bénin, Lomé (Rép. du Togo).

(**) Departamento de Zootecnia UNESP, Campus de Botucatu, 18600 Botucatu, S. P. (Brazil). de l'installation du pâturage compte, entre autres, les frais de défrichement et du labour du terrain, des semences, des engrais, du nettoyage mécanique ou par des herbicides, et parfois de l'irrigation, sans oublier le temps de travail de l'exploitant ou de l'éleveur, très souvent non comptabilisé dans la fixation du coût des produits agricoles. La somme de tous ces éléments du coût de production des fourrages, si tous les moyens sus-mentionnés étaient utilisés, ferait élever considérablement le prix du kilogramme de viande.

Il existerait toutefois quelques possibilités d'installation d'un pâturage en réduisant les frais. Il s'agirait, à ce propos, d'utiliser en période active de végétation (saison des pluies) un sol défriché, labouré, fumé et enrichi aux engrais, pour installer des cultures saisonnières de 3 à 4 mois telles que le mais, le haricot, l'arachide, le riz, etc.... puis en arrière-saison (fin de la saison des pluies), de reprendre le même sol, à la récolte, pour 
l'installation de plantes fourragères, qui bénéficieront alors des dernières pluies et des précédents de fumure et de fertilisation. Le procédé ainsi décrit aurait pour avantage de faire supporter les frais de préparation du terrain, de fumure et de fertilisation du sol aux deux groupes de plantes : les vivrières et les fourragères, et non plus aux seules plantes fourragères. Mais la saison des pluies étant alors avancée, les dernières à occuper le sol, c'est-à-dire les plantes fourragères, risqueraient de voir leur établissement handicapé par la sécheresse très prochaine. Dans le cas d'un établissement acceptable au contraire, les premières pluies de la saison suivante assureraient à ces plantes un meilleur départ avec la possibilité d'une explosion.

Afin de s'assurer des possibilités de réussite du procédé, le présent travail s'est proposé d'étudier le pouvoir d'établissement de quatre graminées tropicales couramment utilisées en production de fourrages, mises en place en arrière-saison. En raison de certaines contraintes de temps, l'essai a été installé sur une jachère, sans aucun apport au sol, en partant de l'idée que si cela réussissait, l'application du procédé conformément à l'idée de base aboutirait, a priori, à des résultats bien plus satisfaisants.

Des observations ont été donc effectuées sur la «prise » de leurs boutures, sur leur comportement végétatif vis-à-vis de la sécheresse, et enfin sur leur pouvoir à s'établir en ce moment, et ce par le biais de leurs productions respectives à la " coupe de formation».

\section{MATÉRIEL ET MÉTHODE}

Cet essai a été réalisé à la Station Expérimentale «Président Médeci », domaine appartenant à l'Universidade Estadual Paulista «Julho de Mesquita Filho "-Campus de Botucatu (Etat de São Paulo, Brésil).

Les coordonnées géographiques de la station sont les suivantes: $22^{\circ} 52$ de latitude Sud, 435 à $810 \mathrm{~m}$ d'altitude, avec une saison pluvieuse, chaude, s'étalant de la mi-octobre à la mi-mai et une saison sèche, froide, de la mi-mai à la mi-octobre; la moyenne thermique annuelle de la localité est de $20^{\circ} \mathrm{C}$ avec une moyenne pluviométrique de $1200 \mathrm{~m}$.

Les espèces végétales utilisées étaient:

- Pennisetum purpureum Schum. var. Vruckwona,

\section{- Panicum maximum Jacq., \\ - Brachiaria decumbens Stapf., \\ - Digitaria decumbens Stent.}

Chacune de ces espèces occupait une aire végétale comportant quatre blocs de sept parcelles, soit vingt-huit parcelles de $6 \mathrm{~m}$ sur $4 \mathrm{~m}$. Les parcelles de chaque aire étaient distantes de $2 \mathrm{~m}$ les unes des autres, et des couloirs de $4 \mathrm{~m}$ séparaient une aire de l'autre.

Le terrain, avant l'établissement des essais, était couvert d'une broussaille de 80 à $120 \mathrm{~cm}$ environ de hauteur composée essentiellement de Panicum maximum, var. "Sempre verde", Cida sp., Brachiaria plantaginea, Cyperus rotondus, Cynodon sp., Melinis minutiflora et Brachiaria mutica. Il a été anciennement exploité (6) pour la production de céréales.

La préparation du terrain d'essai a consisté en un labour de 15 à $20 \mathrm{~cm}$ de profondeur au tracteur, suivi d'un passage de disques.

Le sol, dans le lit supérieur du ruisseau « Lavapés », est d'origine alluviale récente ; il est en conséquence peu évolué et relativement pauvre. Ses caractéristiques chimiques entre 0 et $40 \mathrm{~cm}$ de profondeur étaient : $\mathrm{pH}: 5,65 ; \mathrm{M} . \mathrm{O}: 0,85$ et en $\mathrm{m}$. éq. $\mathrm{g}$ pour $100 \mathrm{~g}$ de terre séchée à l'air, $\mathrm{S}: 2,46 ; \mathbf{T}: 4,78 ; \mathrm{H}^{+}: 2,64 ; \mathrm{Al}^{3+}: 0,28$; $\mathrm{K}^{+}: 0,37 ; \mathrm{PO}^{3-}: 0,50 ; \mathrm{Ca}^{2+}: 1,68$ et $\mathrm{Mg}^{2+}: 0,68$. De la mise en place des plantes à la "coupe de formation » ce sol n'a reçu aucun apport de fumier ni d'engrais.

Il a été utilisé, pour l'installation des essais, des boutures disposées en lignes. L'espacement entre les lignes était de $50 \mathrm{~cm}$ pour Panicum, Brachiaria et Digitaria, et de $70 \mathrm{~cm}$ pour Pennisetum; quant à l'intervalle entre les pieds sur la même ligne, il était de $50 \mathrm{~cm}$ pour Panicum, les trois autres espèces étant plantées, chaumes entiers ou stolons dans des sillons de $15 \mathrm{~cm}$ environ de profondeur.

La plantation eut lieu du 22 mars 1977 et la « coupe de formation », le 08.11.1977. Des observations morpho-phénologiques (port, hauteur de pousse ou longueur de stolons, et mise à fleur) ont été effectuées. Des estimations moyennes de recouvrement ont été également faites à vue d'œil à partir des estimations sur toutes les vingt-huit parcelles de chaque espèce.

Les pouvoirs d'établissement ont été chiffrés à travers la production de chaque espèce à la coupe dite de «formation ». Pour ce faire, il a été pesé, au hasard, les productions de trois parcelles par bloc et par espèce, soit en tout douze parcelles par espèce. Des échantillons 
TABLEAU $\mathrm{N}^{*} \mathrm{I}$ - Données climariques de station.

\begin{tabular}{|c|c|c|c|c|c|}
\hline \multirow{2}{*}{$\begin{array}{c}\text { Mois } \\
\text { (Année 1977) }\end{array}$} & \multirow{2}{*}{$\begin{array}{l}\text { Précipitations } \\
\text { (en m) }\end{array}$} & \multirow{2}{*}{$\begin{array}{c}\text { Température } \\
\left.\text { (en }{ }^{*} \mathrm{C}\right)\end{array}$} & \multicolumn{3}{|c|}{ *Bilan hydrique (en mo) } \\
\hline & & & Excédent & Disponible & Dëficience \\
\hline Mars & 171 & $.23,4$ & 31 & & \\
\hline Avril & 112 & 19,5 & 42 & & \\
\hline Maij: & $\underline{21}$ & 18,2 & & 62 & . \\
\hline Juin & 66 & 17,2 & & 79 & \\
\hline Juillet:* & 30 & $\underline{19,7}$ & & 40 & \\
\hline Août:\%: & $\underline{6}$ & 19,3 & & & $\underline{23}$ \\
\hline Septembre & 89 & 19,8 & & 15 & \\
\hline Octobre & 98 & 21,5 & & 17 & \\
\hline Novembre & 169 & 20,6 & & 98 & . \\
\hline
\end{tabular}

" Bilan hydr ique calculé selon Thornthwaite (1955)

※: Mois secs i.e. où $\mathrm{P} \leqslant 2 \mathrm{~T}$, selon le principe de l'établıssement du diagranme, ombrothermique.

ont été ensuite tirés en vue de la détermination, au laboratoire, du taux moyen de matière sèche de chacune des quatre espèces végétales.

Enfin, des données climatiques de la station ont permis d'envisager l'importance de l'influence de la température et de la disponibilité de l'eau du sol sur l'établissement des plantes.

\section{RÉSULTATS ET DISCUSSION}

\subsection{Les données climatiques}

Le tableau $n^{\circ} \mathrm{I}$ fait état des facteurs du climat qui influenceraient le plus la «prise » des boutures et la croissance des plantes installées.

Les précipitations ont fléchi de $171 \mathrm{~mm}$ en mars pour tomber à $6 \mathrm{~mm}$ en août avant de remonter à $169 \mathrm{~mm}$ en novembre; quant à la température, elle est allée de $23,4^{\circ} \mathrm{C}$ en mars pour atteindre son minimum $17,2^{\circ} \mathrm{C}$ en juin et remonter progressivement jusqu'à $20,6^{\circ} \mathrm{C}$ en novembre.

Le bilan hydrique, qui tient compte de la température, des précipitations et de l'évapotranspiration potentielle a montré que, dans les conditions de ce travail, les plantes en question ont subi un stress hydrique en août, mois où le bilan hydrique de la station a accusé une déficience de $23 \mathrm{~mm}$ d'eau dans le sol.

\subsection{Aspects végétatif et phénologique du pou- voir d'établissement}

Les observations sur l'état végétatif et phénologique des quatre espèces fourragères concernées pendant la période d'établissement se trouvent résumées au tableau $n^{\circ}$ II.

\subsubsection{La «prise » des boutures}

L'estimation de la "prise" des boutures a été faite par observation visuelle, et cette « prise » a été en raison inverse du remplacement des boutures.

Elle a donc été excellente pour Pennisetum et Panicum avec 95 à 98 p. 100 soit encore 2 à 5 p. 100 seulement de remplacement des boutures. Brachiaria a eu une "prise" bonne de 47 à 55 p. 100 à la première plantation, parce qu'il aurait été utilisé à cette occasion des boutures trop âgées; elle a été bonne pour les boutures de remplacement. Quant à Digitaria, ses boutures, ayant séjourné à l'air 2 jours avant leur mise en place, ont eu une prise médiocre de 30 à 32 p. 100 : ses stolons, plus fins, ont dû sécher partiellement avant leur mise en terre.

\subsubsection{Concurrence avec les mauvaises herbes}

La concurrence des graminées plantées avec les mauvaises herbes a été en fonction directe de la vigueur des boutures à prendre et de la nature des mauvaises herbes; cette concurrence a déterminé le nombre de nettoyages opérés au cours de la période d'établissement.

D'une façon générale, les graminées au port érigé, c'est-à-dire Pennisetum et Panicum, se sont montrées les plus concurrentes, probablement par leur ombrage, sur les mauvaises herbes; celles-ci ont demandé un et deux nettoyages respectivement pour Pennisetum et Panicum. Brachiaria et Digitaria ont demandé trois nettoyages. Il convient de faire remarquer, par ailleurs, que le sol, pauvre comme il l'était, constituait un milieu relativement spécialisé où les mieux adaptées étaient les mauvaises herbes. 
TABL. $N^{\circ}$ II $\rightarrow$ Pouvoir d'établissement de 4 graminées fourragères : états végétatif et phénologique.

\begin{tabular}{|c|c|c|c|c|}
\hline & Pennisetum & Paniaum & Brachiaria & Digitaria \\
\hline $\begin{array}{l}\text { 'Prise'des } \\
\text { boutures }\end{array}$ & $\begin{array}{l}\text { bonne }: 2 \text { p.100 envi- } \\
\text { ron de remplacement }\end{array}$ & $\begin{array}{l}\text { bonne : } 5 \text { p.100 env. } \\
\text { de remplacement }\end{array}$ & $\begin{array}{l}\text { moyenne: } 47 \text { p, } 100 \\
\text { env. de remplace- } \\
\text { ment. }\end{array}$ & $\begin{array}{l}\text { médiocre : } 69 \text { p.100. } \\
\text { env. de remplacement }\end{array}$ \\
\hline Mauvaises herbes & $\begin{array}{l}\text { Cynodon sp. } \\
\text { Cida sp. }\end{array}$ & Cyperus rotondus & $\begin{array}{l}\text { Brachiaria pianta- } \\
\text { ginea } \\
\text { Cida sp. }\end{array}$ & $\begin{array}{l}\text { Cyperus rotondus, } \\
\text { Brachiaria ptanta- } \\
\text { ginea - Cida sp. } \\
\text { Cynodon sp. }\end{array}$ \\
\hline $\begin{array}{l}\text { Nombre de } \\
\text { nettoyages }\end{array}$ & 1 & 2 & 3 & 3 \\
\hline \multirow{2}{*}{$\begin{array}{ll} & -27.07 .77 \\
\text { Hauteurs de } & \\
\text { pousse } & -08.11 .77\end{array}$} & $\begin{array}{c}67 \\
(35-105)\end{array}$ & $\left(1.7^{31}-65\right)$ & $\left(12^{17}-37\right)$ & $\left(6^{8}-15\right)$ \\
\hline & $(135-242)$ & $\begin{array}{c}1,63 \\
(104-184)\end{array}$ & $\begin{array}{l}(46-72) \\
\text { rejets longs de } \\
45 \text { à } 70 \mathrm{~cm}\end{array}$ & $\begin{array}{l}\quad 17 \\
\quad\left(7^{-23)}\right. \\
\text { rejets longs de } \\
75-105 \mathrm{~cm}\end{array}$ \\
\hline Port et vigueur & $\begin{array}{l}\text { chaumes grêles et } \\
\text { étalés en coussinet }\end{array}$ & $\begin{array}{l}\text { chaumes grêles et } \\
\text { étalés en coussinet }\end{array}$ & en coussinet & $\begin{array}{l}\text { souffreteux : } \\
\text { cochenilles }\end{array}$ \\
\hline Mise à fleurs & non observêe & $\begin{array}{l}\text { fleurs sur rejets } \\
\text { de } 35-45 \mathrm{~cm} \text { ( } 70-80 \\
\text { p. } 100 \text { des rejets) }\end{array}$ & $\begin{array}{l}\text { fleurs sur des } \\
\text { rejets de } 17-25 \\
\mathrm{~cm}(45-50 \text { p. } 100 \\
\text { des rejets })\end{array}$ & pas encore amorcéc \\
\hline
\end{tabular}

\subsubsection{Vigueur de pousse et port}

D’une façon générale, après la «prise » des boutures, la pousse n'a été vigoureuse pour aucune des quatre espèces plantées. Les chaumes de Pennisetum et de Panicum qui, en situation normale, se trouveraient dressés verticalement avec des hauteurs de pousse bien supérieures, formaient un angle inférieur à $45^{\circ} \mathrm{C}$ avec le sol comme pour le couvrir contre l'évaporation ; en outre, ces deux espèces portaient des feuilles étroites, évitant ainsi une transpiration importante; et ces deux phénomènes donnaient à la plante tout entière l'aspect d'un coussinet. Brachiaria a présenté également un aspect identique; Digitaria apparaissait souffreteux, car abritait au surplus des cochenilles.

\subsubsection{Mise à fleur}

Panicum et Brachiaria ont, après la "prise » de leurs boutures, émis des fleurs sur des chaumes ayant atteint une certaine taille : $40 \mathrm{~cm}$ environ pour Panicum et $20 \mathrm{~cm}$ environ pour Brachiaria. Il n'a pas été observé des fleurs ni chez Pennisetum ni chez Digitaria. En effet, Pennisetum purpureum var. Vruckwona passe pour une plante sans fleurs et Digitaria decumbens, pour une espèce précoce.

La présence ou l'absence de la mise à fleurs ne paraît pas avoir d'influence majeure sur l'état végétatif ni sur la vigueur de pousse des plantes en question ; l'humidité du sol et la température ont semblé en être, au contraire, les facteurs les plus déterminants.

\subsection{Aspects de la production}

Le pouvoir d'établissement d'une plante, outre son expression par le pourcentage de boutures ayant pris, peut également s'estimer par la productivité de la partie aérienne de la plante concernée pendant sa période d'établissement (8), cette productivité traduit la croissance pondérale, c'est-à-dire la vigueur de pousse de la plante en question.

Au bout de 228 jours, soit 32 semaines et demie de prise et de croissance, les productions (tabl. $\mathrm{n}^{\circ}$ III) s'élevaient à $15,48 \pm 1,06 \mathrm{t} / \mathrm{ha}$ et $6,57 \pm 0,58 \mathrm{t} / \mathrm{ha} ; 6,80 \pm 0,57 \mathrm{t} / \mathrm{ha}$ et $0,85 \pm 0,16$ t/ha respectivement pour Pennisetum, Panicum, Brachiaria et Digitaria. Le test de TUKEY à 1 p. 100 de probabilité a présenté entre les valeurs trouvées une différence minimale significative de $3,85 \mathrm{t} / \mathrm{ha}$. Il n'y eut donc pas de différence significative entre les productions de Panicum et Brachiaria ; Pennisetum a eu une production statistiquement supérieure à celles de Panicum et Brachiaria; et les productions de ces deux dernières espèces surpassaient largement celle de Digitaria. 
TABL. N'III - Pouvoir d'établissement de 4 graminées fourragères : aspects de la production.

\begin{tabular}{|c|c|c|c|c|c|}
\hline \multirow{2}{*}{\multicolumn{2}{|c|}{$\begin{array}{l}\text { Taux de matière sèche } \\
\text { à } 65^{\circ} \mathrm{C} \text { (en p.100) }\end{array}$}} & Penisetum & Ponicum & Brachiaria & Digitaria \\
\hline & & $\underline{26,65}$ & $\underline{33,09}$ & $\underline{28,66}$ & 33,16 \\
\hline \multirow{4}{*}{$\begin{array}{l}\text { Productions moyennes } \\
\text { (en tonnes de MS à } \\
65^{\circ} \mathrm{C} \text { par hectare) }\end{array}$} & Bloc $1 \%$ & $18,3 B$ & 5,24 & 6,55 & $-0,54$ \\
\hline & Bloc 2 & 15,68 & 7,31 & 8.49 & 0,69 \\
\hline & Bloc 3 & 14,10 & 7,78 & 6,21 & 0,88 \\
\hline & Bloc 4 & 13,77 & 5,97 & 5,97 & 1,29 \\
\hline \multicolumn{2}{|l|}{$\begin{array}{l}\text { Production moyennew: } \\
\text { par espèce (tonnes } \\
\text { de MS/ha }\end{array}$} & $15,48^{a} \pm 1,06$ & $6,57^{b} \div 0,5 B$ & $6,80^{b} \pm 0,57$ & $0,85^{c} \pm 0,16$ \\
\hline \multicolumn{2}{|l|}{$\begin{array}{l}\text { Coefficient de } \\
\text { variation (en p.100) }\end{array}$} & 13,76 & 17,81 & 16,90 & 11,76 \\
\hline \multicolumn{6}{|c|}{$\begin{array}{l}\text { Test F de TUKEY : significatif à } 1 \text { p.100 de probabilité, avec une différence } \\
\text { minimale significative (DM. S.) de } 3,85 \text {. }\end{array}$} \\
\hline \multicolumn{2}{|l|}{$\begin{array}{l}\text { Production primaire } \\
\text { nette de la përiode } \\
\left(\text { en } \mathrm{g} / \mathrm{m}^{2} / \mathrm{j} .\right)\end{array}$} & 6,70 & 2,88 & 2,98 & 0,37 \\
\hline
\end{tabular}

* Moyenne de 3 parcelles du même bloc. "W Comparaison entre les 4 espèces : les productions portant la même lettre en indice sont statistiquement équivalentes avec une probabilité de 99 p.100.

Rectificatif : $1^{\text {ro }}$ colonne, $11^{\text {e }}$ ligne : supprimer $"$ de TUKEY .

$1^{\text {te }}$ colonne, 12e ligne : lire (PPDS) au lieu de (DM. S.); ajouter après 3,85: (test de TUKEY à 1 p. 100).

Il convient de faire remarquer enfin que les valeurs aussi faibles obtenues pour Digitaria seraient en grande partie imputables à l'attaque de cette espèce végétale par des Cochenilles (Antonina graminis, Maskell). En effet, on pouvait observer aux faces inférieures des stolons et des feuilles des amas blanchâtres farineux et doux au toucher : ce sont des adultes et larves dans leurs sacs ovigères. FONSECA (2) a mentionné Digitaria decumbens en tête des espèces fourragères plus attaquées, et Pennisetum purpureum, Panicum maximum et Brachiaria decumbens comme des moins attaquées. NUTI et LUCCI, 1969 cités par SARTINI (5), ont estimé que les Cochenilles pouvaient provoquer des pertes de productions allant de 18 p. 100 pour Chloris gayana à 59 p. 100 pour Cynodon dactylon.

Les valeurs moyennes des «productivités primaires nettes aériennes " (en g de M. S. $/ \mathrm{m}^{2} / \mathrm{j}$ ) des quatre espèces graminéennes au cours de leur période d'établissement étaient de $6,79 \mathrm{~g} / \mathrm{m}^{2} / \mathrm{j} ; 2,88 \mathrm{~g} / \mathrm{m}^{2} / \mathrm{j} ; 2,98 / \mathrm{m}^{2} / \mathrm{j}$ et $0,37 \mathrm{~g} / \mathrm{m}^{2} / \mathrm{j}$ respectivement pour Pennisetum, Panicum, Brachiaria et Digitaria. ANDRADE et GOMIDE (1) ont trouvé à Viçosa (Brésil) en période active de végétation $22,99 \mathrm{~g} / \mathrm{m}^{2} / \mathrm{j}$ pour Pennisetum purpureum, var. «Taiwan-A-146»; PEDREI-
RA (4), a trouvé en période de végétation active à Nova Odessa (Brésil) $11,64 \mathrm{~g} / \mathrm{m}^{2} / \mathrm{j}$ pour $P$ anicum maximum pendant $187 \mathrm{j}$ de croissance ; VERGUEZ (7) a trouvé en arrière-saison (23 août7 octobre) au Venezuela $3,7 \mathrm{~g} / \mathrm{m}^{2} / \mathrm{j}$ pour Digitaria decumbens après 45 jours de croissance ; enfin, KPAKOTE et al. (3) ont enregistré en période de végétation active (1977/78) à Botucatu respectivement avec ou sans engrais, les valeurs de 17,43 contre $11,41 \mathrm{~g} / \mathrm{m}^{2} / \mathrm{j}$ pour $P$ ennisetum purpureum var. Vruckwona, 11,32 contre $12,98 \mathrm{~g} / \mathrm{m}^{2} / \mathrm{j}$ pour Panicum maximum, 11,47 contre $11,44 \mathrm{~g} / \mathrm{m}^{2} / \mathrm{j}$ pour Brachiaria decumbens et 2,00 contre $2,56 \mathrm{~g} / \mathrm{m}^{2} / \mathrm{j}$ pour Digitaria decumbens, toutes sur 196 jours de croissance.

Il ressort de ce qui précède que les " productivités primaires nettes aériennes " seraient influencées par la richesse du sol surtout pour le Pennisetum et le Digitaria, et par les facteurs climatiques tels que la longueur des jours, l'ensoleillement, la température et les précipitations; ces facteurs climatiques seraient les premiers responsables des différences rencontrées entre les " productivités primaires nettes aériennes " de la période allant de la fin de la saison des pluies au début de la saison des pluies et celles de la période de végétation active c'est-àdire de la saison des pluies. 


\section{CONCLUSIONS}

Le présent travail a pour objectif de juger de la faisabilité de l'implantation d'herbage en arrière-saison, et derrière une culture saisonnière. Il appelle les conclusions suivantes :

- Dans les conditions pratiquement défavorables décrites plus haut, le procédé s'est montré réalisable, sauf peut-être pour Digitaria dont les résultats insatisfaisants seraient plutôt dus à une attaque par des cochenilles;

- La série de désherbages effectués lors de l'essai s'expliquerait par l'utilisation d'un terrain de jachère ; dans la conformité au procédé, il n'en aurait fallu qu'un seul, étant donné tous les soins exigés par les cultures vivrières precédentes ;

- Un épandage de fumier et une application d'engrais de fond auraient amélioré encore les conditions de ce sol pauvre, en élevant son taux de matière organique et sa concentration en éléments minéraux essentiels ;

- Une irrigation d'appoint en juillet et surtout en août aurait favorisé un meilleur établissement ;

- Ce procédé de culture « indirecte » de plantes fourragères aurait de grandes chances d'être accepté des cultivateurs, puisqu'il ne demande pas de gros moyens financiers sinon ceux seulement exigés pratiquement par la culture vivrière, en tête.

\section{REMERCIEMENTS}

Les auteurs expriment leur reconnaissance au Gouvernement Fédéral du Brésil qui a rendu ce travail possible, au Rectorat de l'Universidade Estadual Paulista «Julho de Mesquita Filho », aux Responsables du Campus de Botucatu, de la Faculté de Médecine Vétérinaire et Zootechnique et du Département de Zootechnie pour les moyens mis à leur disposition.

Leurs remerciements vont également à la direction de la Station Expérimentale "Presidente Médeci » du Campus de Botucatu et au personnel de terrain de ladite station.

\section{SUMMARY}

Possibility of shooting of four graminaceous tropical fodders

(Pennisetum purpureum Schum., Panicum maximum Jacq., Brachiaria decumbens Stapf., et Digitaria decumbens Stent.) planted at the end of the rainy season

Morphological, phenologic as well as ponderal studies have been effected on the possibility of shooting and growth of four graminaceous tropical fodders (Pennisetum purpureum, Panicum maximum, Brachiaria decumbens and Digitaria decumbens) planted at the end of the rainy season at Botucatu, São Paulo State (Brazil) in a soll of alluvial origin, not very much mature, and of relatively poor quality. The studies extended over a period of nearly thirtytwo and half weeks $i$. e. from March ending 1977 to the beginning of November of the same year.

The performance of young shoots sliced for reproduction has been excellent for Pennisetum and Panicum (about 95 to 98 p. 100), average for Brachiaria (about 53 p. 100) and mediocre for Digitaria (about $42 \mathrm{p.} \mathrm{100)}$ ). The number of weedings has varied according to species : one for Pennisetum, two for Panicum, and three for Brachiaria and Digitaria. The average dry matter produced during this period of formation ranged from $15.48 \pm 1.06 \mathrm{t} / \mathrm{ha}$ for Pennisetum, $6.57 \pm 0.58$ t/ha for Panicum, $6.80 \pm 0.57$ t/ha for Brachiaria to $0.85 \pm 0.16 \mathrm{t} / \mathrm{ha}$ for Digitaria. No significant differences have been registered between the average productions of Panicum and Brachiaria by the application of Tukey test at 1 p. 100 and 5 p. 100 probability levels.

The poor results obtained for Digitaria could be imputed to the intense infestation of the species by Mealybugs.

These studies showed also in Botucatu conditions, the possibility of pasture setting up in late season and behind a seasonal crop, method which would bring a good deal of advantages.

\section{RESUMEN}

Possibilidades de desarrollo de cuatro gramineas forajeras tropicales (Pennisetum purpureum Schum., Panicum maximum Jacq., Brachiaria decumbens Stapf., y Digiraria decumbens Stend.) plantadas al fin de la estación lluviosa

Se hicieron observaciones morfológicas, fenolbgicas y ponderales sobre el desarrollo de cuatro gramíneas forajeras tropicales (Pennisetum purpureum, 
Panicum maximum, Brachiaria decumbens y Digitaria decumbens) plantadas al fin de la estación lluviosa en Botucatu, Estado de São Paulo (Brazil).

Dichas observaciones duraron desde el fin de marzo hasta el principio de noviembre de 1977 , sea cerca de 32 semanas y media. El suelo utilizado era de origen aluvial, poco evolucionado y relativamente pobre.

Las estacas de Pennisetum y de Panicum agarraron de modo excelente (cerca de 95 a 98 p. 100), las de Brachiaria medianamente (cerca de 53 p. 100) $\mathrm{y}$ las de Digitaria mediocremente (cerca de 31 p. 100).

El número de escardaduras varió según las especies, sea : uno para Pennisetum, dos para Panicum y tres para Brachiaria y Digitaria. Las producciones medias a la corta de formación expresas sobre el base de la materia seca a $65^{\circ} \mathrm{C}$ eran de $15,48 \pm 1,06 \mathrm{t} /$ ha para Pennisetum, $6,57 \pm 0,58$ t/ha para Panicum, $6,80=0,57$ t/ha para Brachiaria y $0,85 \pm 0,16 \mathrm{t} /$ ha para Digitaria; la prueba de Tukey a 1 p. 100 y 5 p. 100 de probabilidad no mostró diferencias significativas entre las producciones medias de Panicum y de Brachiaria. Los malos resultados obtenidos serian imputables a la infestación intensa de dicha especie por cochinillas.

Estas observaciones mostraron, por otro lado, que, en las condiciones de Botucatu, la posibilidad de instalación de un pasto al fin de la estación lluviosa y después de un cultivo estacional es un método teniendo muchas ventajas.

\section{RESUMO}

Velocidade de estabelecimento de quatro gramineas forrageiras tropicais (Pennisetum purpureum Schum., Panicum maximum Jacq., Brachioria decumbens Stapf. et Digitaria decumbens Stent.) instalados no finado da estacão chuvosa

Observaçōes morfológıcas, fenológicas e ponderais têm sido efetuadas sôbre a velocidade de estabelecimento de quatro Gramineas forrageiras tropicais (Pennisetum purpureum, Panicum maximum, Brachiaria decumbens e Digitaria decumbens) instaladas no fim da estação chuvosa em Botucatu, Estado de São Paulo (Brasil). Observaçōes estas foram do fim de Março de 1977 ao inicio de Novembro do mesmo ano, seja perto de 32 semanas e meia. $O$ solo usado para a instalação do ensaio é de origem aluvial, pouco evoluido e relativamente pôbre.

A brotação das mudas tem s1do excelente (95 a 98 p. 100 aproximadamente) para Pennisetum e Pancum, média (53 p. 100 aproximadamente) e mediocre para Brachiaria e Digiraria (31 p. 100 aproximadamente). $\mathbf{O}$ número de capinas variou segundo as especies seja : uma para Pennisetum, duas para Panicum e três para Brachiaria e Digitaria. As produçōes médias ao « corte de formação ", expressas na base da matéria seca a $65^{\circ} \mathrm{C}$, eram de $15,48 \pm 1,06 \mathrm{t} / \mathrm{ha}$ para Pennisetum, 6, $57 \pm 0,58$ t/ha para Panicum, 6,80 $\pm 0,57$ t/ha para Brachiaria e $0,85=0,16$ t/ha para Digitaria; o test de Tukey a 1 p. 100 e a 5 p. 100 de probabilidade não deu diferenças significativas entre as producões médias de Panicum e Brachiaria.

Os fracos resultados obtidos para Digitaria seriam devidos à infestação desta especie por Cochonilhas.

Estas observaçōes têm mostrado em condiçōes de Botucatu, alias, a possibilidade de instalação de um pasto no finado da estação chuvosa e atrás de uma cultura estacional, pratica que trana consigo muitas vantagens.

\section{BIBLIOGRAPHIE}

1. ANDRADE (I. F.), GOMIDE (J. A.). Curva de crescimento e valor nutritivo do Capim-elefante (Pennisetum purpureum Schum.) "A-146-Taiwan". Rev. CERES, Viçosa-M. G., 1971, 18 (100): 431-447.

2. FONSECA (J. P.). Uma cochonilha recentemente introduzida no Brasil. Biólogo, 1967, $33(3)$ : 57-61.

3. KPAKOTE (K. G.) et al. Travail non publié, 1978.

4. PEDREIRA (J. V. S.). Estudo de crescimento do Capim-Coloniâo (Panicum maximum, Jacq.). Bol. Indústr. Anim., São Paulo, 1965-66, 26 (No único) : 263-273.

5. SARTINI (H. J.). Problemas de infestaçảo de cocho- nilhas nos pastos. Zootecnia, Nova Odessa-SP., 1969 , 7 (4) : 25-42.

6. SILVEIRA (A. C.). Contribuição para o estudo do capım elefante (Pennisetum purpureum, Schum.) como reserva forrageira no tropico-Tése de Docência livre, Botucatu-SP, 1976, 224 p.

7. VERGUEZ (O. G.). Crescimento de pasto estrella y pasto pangola In : $9^{\circ}$ Congresso Internacional de Pastagens, São Paulo, 1965, Vol. 1, p. 443-8.

8. WERNER (J. C.) er al. Velocidade de estabelecimento e produção de feno de dez leguminosas forrageiras e do capim-gordura. Bol. Indústr. anim., Sāo Paulo, 1975, 32 (2) : 331-345. 